\title{
Chimeric antigen receptor T-cell therapy for multiple myeloma: a consensus statement from The European Myeloma Network
}

Haematologica 2019

Volume 104(12):2358-2360

\section{Correspondence: \\ PHILIPPE MOREAU \\ philippe.moreau@chu-nantes.fr \\ Received: April 24, 2019. \\ Accepted: August 21, 2019. \\ Pre-published: August 22, 2019.}

doi:10.3324/haematol.2019.224204

Check the online version for the most updated information on this article, online supplements, and information on authorship \& disclosures: www.haematologica.org/content/104/12/2358

(C)2019 Ferrata Storti Foundation

Material published in Haematologica is covered by copyright. All rights are reserved to the Ferrata Storti Foundation. Use of published material is allowed under the following terms and conditions:

https://creativecommons.org/licenses/by-nc/4.0/legalcode. Copies of published material are allowed for personal or internal use. Sharing published material for non-commercial purposes is subject to the following conditions:

https://creativecommons.org/licenses/by-nc/4.0/legalcode, sect. 3. Reproducing and sharing published material for commercial purposes is not allowed without permission in writing from the publisher.

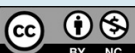

Philippe Moreau, ${ }^{1}$ Pieter Sonneveld, ${ }^{2}$ Mario Boccadoro, ${ }^{3}$ Gordon Cook, ${ }^{4} \mathrm{Ma}$ Victoria Mateos, ${ }^{5}$ Hareth Nahi, ${ }^{6}$ Hartmut Goldschmidt, ${ }^{7}$ Meletios A. Dimopoulos, ${ }^{8}$ Paulo Lucio, ${ }^{9}$ Joan Bladé, ${ }^{10}$ Michel Delforge,${ }^{11}$ Roman Hajek, ${ }^{12}$ Heinz Ludwig, ${ }^{13}$ Thierry Facon, ${ }^{14}$ Jesus F. San Miguel ${ }^{15}$ and Hermann Einsele ${ }^{16}$

${ }^{1}$ University Hospital Hôtel-Dieu, Nantes, France; ${ }^{2}$ Erasmus Medical Center, Rotterdam, the Netherlands; ${ }^{3}$ Università di Torino/Azienda Ospedaliera San Giovanni, Torino, taly; ${ }^{4}$ Leeds Institute of Cancer and Pathology, University of Leeds, Leeds, UK; ${ }^{5}$ University of Salamanca, Salamanca, Spain; ${ }^{\circ}$ Karolinska Institutet, Stockholm, Sweden; ${ }^{7}$ Universitätsklinikum Heidelberg, Heidelberg, Germany; ${ }^{8}$ University Athens School of Medicine, Athens, Greece; ${ }^{~} \mathrm{Fundação} \mathrm{Champalimaud,} \mathrm{Lisbon,} \mathrm{Portugal;}{ }^{10} \mathrm{Hospital}$ Clinic de Barcelona, Barcelona, Spain; ${ }^{11}$ Department of Hematology, Catholic University of Leuven, Leuven, Belgium; ${ }^{12}$ University of Ostrava, Ostrava, Czech Republic; ${ }^{13}$ Wilhelminen Cancer Research Institute, Wilhelminen, Austria; ${ }^{14}$ University Hospital Hurriez, Lille, France; ${ }^{15}$ University of Navarra, Navarra, Spain and ${ }^{16}$ University of Wurzburg, Wurzburg, Germany

\begin{abstract}
A doptive cellular therapy using chimeric antigen receptor T-cell (CART) therapy is currently being evaluated in patients with relapsed / refractory multiple myeloma (MM). The majority of CAR-T cell programs now being tested in clinical trials are targeting B-cell maturation antigen. Several recent phase I / II trials show promising preliminary results in patients with MM progressing on proteasome inhibitors, immunomodulatory drugs and monoclonal antibodies targeting CD38. CAR-T cell therapy is a potentially life-threatening strategy that can only be administered in experienced centers. For the moment, CAR-T cell therapy for MM is still experimental, but once this strategy has been approved in relapsed/refractory $\mathrm{MM}$, it will become one of the most important indications for this therapy in Europe and world-wide. This manuscript proposes practical considerations for the use of CAR-T cell therapy in MM, and discusses several important issues for its future development.
\end{abstract}

\section{Introduction}

In recent years, the development of immunotherapy has revolutionized the treatment of cancer, including hematologic malignancies and multiple myeloma (MM). ${ }^{1}$ Therapeutic agents which induce the autologous immune cells to mediate tumor cell killing and to overcome the immunosuppressive mechanisms of the tumor microenvironment may improve clinical outcome. In this setting, adoptive cellular therapy using chimeric antigen receptor (CAR-T), a redirection strategy of $\mathrm{T}$ cells with the goal of increasing the frequency of tumor-directed and functionally active $\mathrm{T}$ cells targeting specifically expressed antigens on myeloma cells, is currently being evaluated in patients with MM. ${ }^{2}$

CAR-T cell therapy has already been approved by the US Food and Drug Administration (FDA) and the European Medicines Agency (EMA) for the treatment of relapsed / refractory B-cell acute lymphoblastic leukemia (B-ALL) in pediatric and young adult patients, and for diffuse large B-cell lymphoma (DLBCL). At the time of writing (2019), it is under evaluation for the treatment of relapsed / refractory MM (RRMM) in phase I / II trials ${ }^{3}$ and phase III trials sponsored by different pharmaceutical companies have just started in these patients. Therefore, CAR-T cell therapy will not have been approved for the treatment of MM by the end of 2019.

B-cell maturation antigen (BCMA, also named TNFRSF17 or CD269) belongs to the family of tumor necrosis factors. It was initially identified on the cell surface of 
the normal and malignant plasma cells and B lymphocytes. ${ }^{4} \mathrm{BCMA}$ is also expressed on myeloma cell lines. ${ }^{5}$ Considering the strong and fairly homogeneous expression of the BCMA receptor on malignant plasma cells and its important mechanism of action, BCMA represent an ideal therapeutic target for CAR-T cell therapy. ${ }^{6}$ Indeed, the majority of CAR-T cell programs currently being tested in clinical trials are targeting BCMA. Other targets on $\mathrm{MM}$ cells are under early preclinical development. The first 'in human' clinical trial of CAR-T cells targeting BCMA that showed anti-myeloma activity was published in 2016.' Several recent phase I / II trials show promising preliminary results in $\mathrm{MM}$ patients progressing on proteasome inhibitors, immunomodulatory drugs (IMiD) and monoclonal antibodies targeting CD38. ${ }^{8-9}$ In this situation of unmet medical need, high response rates and minimal residual disease (MRD) negativity are achieved, and the median progression-free survival (PFS) in responding patients is $>15$ months. ${ }^{5,8,9}$ Nevertheless, no plateau has been observed on PFS curves, indicating the low probability for cure, at least in these heavily pretreated patients. CAR-T cell therapy is a potentially life-threatening strategy that that can only be administered in experienced centers. The major toxicity in MM patients is the cytokine release syndrome (CRS) that may require short hospitalization in intensive care units. ${ }^{5,6,10}$ Neurotoxicity seems far less frequent and severe when compared to CAR-T cell therapy for B-ALL and DLBCL. ${ }^{5-6,10}$ Overall, fewer than 100 patients with RRMM treated with CAR-T cells targeting BCMA have been reported. These patients were highly selected, had refractory disease at the time of entry into clinical trials, while still having good clinical status, and were able to remain without any therapeutic intervention for 4-5 weeks during the manufacturing process of CART cells.

CAR-T cell therapy is by far one of the most expensive therapies for hematologic malignancies.

\section{Practical considerations}

Once CAR-T cell therapy is approved in RRMM, it will become one of the most important indications for this strategy in Europe and world-wide.

For the moment, CAR-T cell therapy for $\mathrm{MM}$ is still experimental..$^{7-8}$ Clinical programs need to be developed under the scientific guidance of the disease experts and cooperative groups in selected centers.

The results of large company-sponsored phase II trials using CAR-T cells infused at the optimal dose are expected at the end of 2019. Several phase III trials have just started (2019), testing CAR-T cells either in very advanced patients or earlier in the course of the disease, especially in high-risk patients. Very few academic trials using CAR-T cell therapy are currently ongoing. The CAR-T cell therapy is different from allogeneic stem cell transplantation (allo-SCT)..$^{5-6}$ There is no scientific reason to support the experience on allo-SCT as the only prerequisite for the identification / selection of centers accredited for CAR-T cell therapy. Toxicity of CAR-T cells is different from that of allo-SCT: the use of a protected environment is not needed, graft-versus-host disease (GvHD) is by definition absent. Given this, one of the most common links between allo-SCT and the CAR-T programs is the apheresis process that will require pertinent accreditations and/or certifications. Experience in hematopoietic transplantation (allo and/or auto) is very important, and expertise in cell therapy manipulation is critical. CAR-T therapy should be performed by a team of experts in $\mathrm{MM}$ and in cellular therapy, which curently includes experts in the specific disease and in autologous and/or allogeneic transplantation, in close collaboration with, among others, intensive care specialists and neurologists, together with a well trained nursing team.

CAR-T cell therapy for MM will potentially become a highly-specialized medication, associated with very high costs. Indications for the use of CAR-T therapy should be clearly defined by experts in $\mathrm{MM}$, if possible through international guidelines. A European consensus, defined by co-operative groups, national societies of hematology, the European Myeloma Network (EMN) and the European Hematology Association (EHA) should be proposed to national and European authorities.

The creation of a list of centers of excellence in each European country, certified for CAR-T cell therapy and other advanced immunotherapies, based on disease area and expertise is of the utmost importance. This list may be drawn up by national societies and national health authorities, in the overarching framework of the main European societies, including the specific myeloma groups in each country as well as the European Myeloma Network and EHA.

A specific European registry for the follow up of patients receiving CAR-T cell therapy including treatment indications, response data, previous and subsequent lines of therapy and treatments, safety and long-term follow up (e.g. to monitor for insertion mutagenesis after genetic modification of $T$ cells) must be compiled. As all data from patients treated with CAR-T cells will be registered on the database of the European Group for Blood and Marrow Transplantation (EBMT), it will be mandatory to build a working group combining myeloma experts and national co-operative groups to ensure the accuracy of the data submitted. The presence of disease experts in this group is key to generate scientific knowledge and to propose new studies. This registry should also collect data from other types of innovative and expensive immunotherapies in order to compare their safety and efficacy.

\section{Important issues in the near future}

CAR-T cell therapy is still an experimental therapy, mainly developed by pharmaceutical companies. Academic research programs are urgently needed to improve efficacy and safety, either working with biotech companies or big pharma or independently. A particular focus should be to improve persistence of CAR-T cells, avoid antigen loss and reduce CRS/neurotoxicity. ${ }^{10,11}$ This is nothing new in myeloma, a disease in which the close collaboration between the pharmaceutical companies and the European co-operative group has led to an improvement in outcomes.

The definition of the optimal use of CAR-T cells, including very precise indications and approval, requires the guidance of myeloma experts. For example, when is a patient to be considered a candidate for CAR-T cell therapy? What is the optimal bridging therapy? Does this also depend on renal function? What are the alternative treatment options? Such questions clearly indicate that the 
development of guidelines is mandatory, especially in the current complex therapeutic landscape of this disease.

It is crucial that priorities be clearly defined. Academic programs must be developed in order to: (i) reduce the high cost of CAR-T cells proposed by pharmaceutical companies; (ii) increase academic knowledge on CAR-T cell therapy; (iii) propose new collaborations with pharmaceutical companies; and (iv) design EU clinical trials based on the combination of CAR-T with current or new anti-myeloma strategies.

Educational programs for the use of CAR-T cells should be developed through the different European societies, such as the EMN, ESH, EHA, international societies like the International Myeloma Society (IMS), and the national societies or co-operative groups.
The definition of consensus guidelines and educational programs for autologous CAR-T cell therapy could be expanded to other immunotherapeutic approaches, such as bispecific antibodies, conjugates, NK-cell therapy or allo-CAR-T. Hopefully, in the near future, several strategies targeting BCMA and other plasma cell antigens will become available for the treatment of myeloma patients. The right time for each myeloma patient to use a CART or a bispecific antibody or antibody drug conjugate should be defined by clinical experts, and this will require in-depth knowledge of the disease. Educational programs under the guidance of myeloma experts will not only help improve the outcome of our patients, but also contribute to a more efficient use of the resources available.

\section{References}

1. Franssen LE, Mutis T, Lokhorst HM, van de Donk NWCJ. Immunotherapy in myeloma: how far have we come? Ther Adv Hematol. 2019;10:2040620718822660.

2. Mikkilineni L, Kochenderfer JN. Chimeric antigen receptor T-cell therapies for multiple myeloma. Blood. 2017;130(24):2594-2602.

3. Shah NN, Maatman T, Hari P, Johnson B. Multi Targeted CAR-T Cell Therapies for B-Cell Malignancies. Front Oncol. 2019;9:146.

4. Laâbi Y, Gras MP, Carbonnel F, et al. A new gene, $\mathrm{BCM}$, on chromosome 16 is fused to the interleukin 2 gene by a $\mathrm{t}(4 ; 16)(\mathrm{q} 26 ; \mathrm{p} 13)$ translocation in a malignant $T$ cell lym- phoma. EMBO J. 1992:11(11):3897-3904

5. Gavriatopoulou M, Ntanasis-Stathopoulos I, Dimopoulos MA, Terpos E. Anti-BCMA antibodies in the future management of multiple myeloma. Expert Rev Anticancer Ther. 2019;19(4):319-326.

6. Cohen AD. CAR T-cell therapy against Bcell maturation antigen in multiple myeloma. Clin Adv Hematol Oncol. 2018;16(12): 804-806.

7. Ali SA, Shi V, Maric I, et al. T cells expressing an anti-B-cell maturation antigen chimeric antigen receptor cause remissions of multiple myeloma. Blood. 2016;128(13): 1688-1700.

8. Raje NS, Berdeja JG, Lin Y, et al. AntiBCMA CAR T-cell therapy bb2121 in relapsed or refractory multiple myeloma. $\mathrm{N}$
Engl J Med. 2019;380(18):1726-1737.

9. Zhao WH, Liu J, Wang BY, et al. Updated analysis of a phase 1, open-label study of LCAR-B38M, a chimeric antigen receptor I cell therapy directed against B-Cell maturation antigen, in patients with relapsed/refractory multiple myeloma. Blood. 2018;132(Suppl 1):955.

10. Lee DW, Santomasso BD, Locke FL, et al ASTCT Consensus Grading for Cytokine Release Syndrome and Neurologic Toxicity Associated with Immune Effector Cells. Biol Blood Marrow Transplant. 2019;25(4): 625-638.

11. Hamieh M, Dobrin A, Cabriolu A, et al. CAR T cell trogocytosis and cooperative killing regulate tumour antigen escape. Nature. 2019;568(7750):112-116. 